\title{
Feiern Blutegel ein Comeback?
}

\author{
Die chronische Epikondylitis ist ein \\ Schmerzsyndrom mit sehr limitierten \\ Therapiemöglichkeiten. Da eine \\ Blutegelbehandlung in früheren Stu- \\ dien positive Effekte bei Knie- und \\ Handarthrosen gezeigt hatte, lag es \\ nahe, die Therapie auch bei der Epi- \\ kondylitis zu probieren.
}

- Deutsche Forscher randomisierten 40 Patienten mit Epikondylitis in zwei Gruppen. Die Experimentalgruppe erhielt eine einzige Behandlung mit zwei bis vier Blutegeln, die im schmerzenden Bereich angesetzt wurden. Die Kontrollgruppe erhielt $\sim 30$ Tage lang topisch appliziertes Diclofenac. Der primäre Studienendpunkt waren die Schmerzen am Tag sieben.

Die Experimentalgruppe gab eine Schmerzreduktion von 48 Punkten an, während sich die Schmerzen in der Kontrollgruppe um 3 Punkte verschlimmerten. Die Lebensqualität zeigte eine nicht signifikante Verbesserung bei den mit Blutegel behandelten Patienten. Die Autoren folgern aus diesen Daten, dass eine einmalige Behandlung mit Blut-

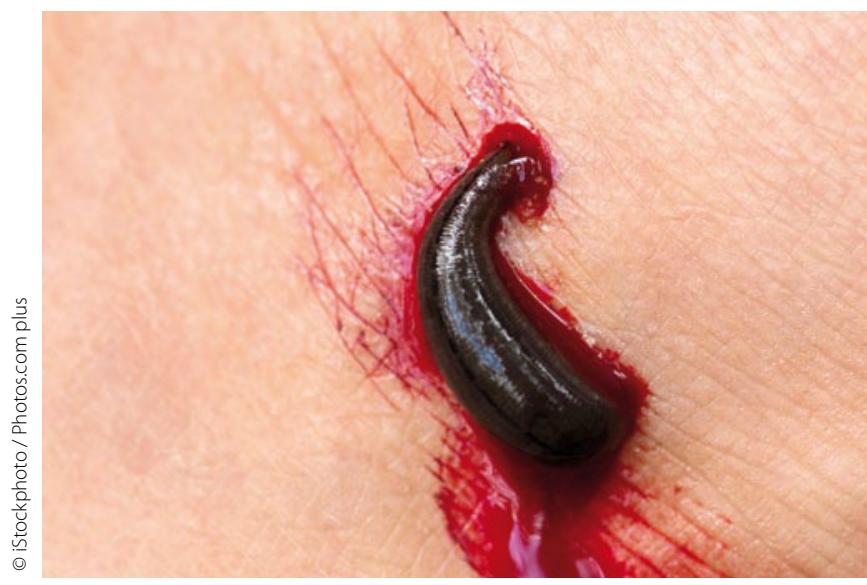

Wie testet man Blutegel gegen Placebo? egeln effektiv sei, und dass die Blutegeltherapie bei dieser Indikation als therapeutische Option in Betracht gezogen werden sollte.

\section{Kommentar}

In der letzten Zeit wurden mehrere Blutegelstudien zu verschiedenen Indikationen publiziert. Allen ist gemeinsam, dass sie keinen ernsthaften Versuch machen, auf Placeboeffekte zu kontrollieren, dass unabhängige Replikationen fehlen, und dass viele Fragen offen bleiben. Wer jemals Blutegel angesetzt hat, weiß, wie beeindruckend diese Prozedur ist. Es ist daher mit einem überdurchschnittlich großen Placeboeffekt zu rechnen. Die vorliegende Studie hat zu viele Schwächen, um sie hier alle zu nennen. Daher ist die Schlussfolgerung der Autoren erstaunlich und die daraus abgeleitete Therapieempfehlung eher realitätsfremdes Wunschdenken als evidenzbasierte Medizin.

E. ERNST :

\section{- M. Bäcker et al.}

Effectiveness of leech therapy in chronic lateral epicondylitis: a randomized controlled trial. Clin. J. Pain. 27 (2011) 442-447

\section{Komasaufen schädigt das Gedächtnis}

\author{
Wer regelmäßig dem Komasaufen - \\ dem sporadischen Konsum großer \\ Alkoholmengen in kurzen Zeitperio- \\ den - nachgeht, hat Probleme, Neues \\ zu lernen.
}

- Wissenschaftler der Universität in Santiago de Compostela (Spanien) haben die Assoziation zwischen Komasaufen und dem verbalen, visuellen und deklarativen Gedächtnis bei Studenten untersucht. 122 Studenten (Alter:18-22 Jahre), 62 Komatrinker (32 Männer und 30 Frauen) und 60 Nichtkomatrinker (31 Männer und 29 Frauen) wurden vier neurophysiologischen Tests unterzogen.
Hauptergebnisse: Die Komatrinker zeigten bei Gedächtnisaufgaben deutlich geringere Leistungen. Das verbale, deklarative Gedächtnis war bei Komatrinkern unabhängig vom Geschlecht schlechter. Sie nahmen neue Informationen schwerer auf, und ihre Studienleis-tungen waren schlechter. Dieser Zusammenhang blieb auch dann bestehen, wenn andere Faktoren in den Berechnungen berücksichtigt wurden.

\section{Kommentar}

Die Befunde stimmen überein mit der Verletzbarkeit des jugendlichen Hippocampus gegenüber den neurotoxischen Effekten des
Alkohols. Beunruhigend ist, dass das Komatrinken nach dem Drogen- und Suchtbericht der Bundestagbeauftragten für das Jahr $2011 \mathrm{im}$ Gegensatz zu allen anderen Drogenmissbräuchen zunimmt und bei 16,7\% der Minderjährigen anzutreffen sein soll.

K. MALBERG =

\footnotetext{
- M. Parada et al.

Binge drinking and declarative memory in university students. Published online on 16 May 2011 in Alcoholism: Clin. Exper. Res.; doi: 10.1111/j.1530-0277.2011.01484.x
} 